\title{
Tabagismo não limita o incremento da força muscular respiratória em pacientes submetidos ao treinamento muscular inspiratório pré-esofagectomia
}

\author{
Smoking doesn't limit the increase in respiratory muscle strength in \\ patients undergoing pre-inspiratory muscle training esophagectomy \\ Marisa de Carvalho Ramos', Taciana Freitas Agrelli', Élida Mara Carneiro², Eduardo Crema ${ }^{3}$
}

RESUMO I Um programa de treinamento muscular respiratório (TMR) para pacientes tabagistas no pré-operatório ambulatorial pode melhorar a força muscular e a capacidade funcional respiratória, evitando complicações que aumentem a permanência do paciente no hospital. O objetivo deste trabalho foi avaliar a eficácia do treinamento muscular inspiratório (TMI) em pacientes tabagistas e não tabagistas que seriam submetidos à cirurgia do megaesôfago. Foram estudados 17 pessoas, divididas em dois grupos: o tabagista (GT), composto por 10 pacientes (58,82\%), e o não tabagista (GNT), com 7 pacientes (41,18\%). A análise dos dados relacionando os dois foi expressa da seguinte forma: os valores de idade e as medidas antropométricas foram comparadas pelo teste $t$ de Student e os valores da pressão inspiratória máxima (PImáx) e da pressão expiratória máxima (PEmáx) pelo teste $t$ de Student pareado. Os dados foram expressos em média \pm desvio-padrão quando verificada a normalidade. Consideraram-se diferenças estatisticamente significativas se p<0,05. Na análise comparativa, observou-se aumento significativo da PImáx após as 4 semanas do TMI, como se segue: PImáx no GT de $-57,20 \pm 18,76$ para $-79,00 \pm 15,38$ e no GNT de $-52,00 \pm 18,76$ para $-72,66 \pm 19,33$. A fisioterapia profilática no pré-operatório ambulatorial proporcionou aumento significativo na força dos músculos inspiratórios em ambos os grupos, evidenciada pelo acréscimo na PImáx com consequente melhora da capacidade ventilatória.

Descritores I fisioterapia; esofagectomia; tabagismo.
ABSTRACT I A program of muscular respiratory training to smoker patients in pre-operatory ambulatory can improve the muscle strength and the functional respiratory capacity, avoiding complications which increase the hospitalization period of the patient. The aim of this work was to evaluate the effectiveness of the inspiratory muscle training in smoker and non-smoker patients who would be submitted to a megaesophagus surgery. Seventeen patients were studied, divided into two groups: smoker (GT), composed of 10 patients (58.82\%), and the non-smoker (GNT), with 7 patients (41.18\%). Data analysis comparing the two groups was expressed as follows: the values of age and anthropometric measurements were compared by Student's t-test and the values of Maximal Inspiratory Pressure (MIP) and Maximal Expiratory Pressure (MEP) were compared by the paired Student's t-test. Data were expressed as mean \pm standard deviation when checked to normal. Differences were considered statistically significant if $p<0.05$. Level of significance adopted was $p=0.05$. Considering the comparative analysis, it was observed a significant increase of Maximal Inspiratory Pressure MIP after the 4 weeks of inspiratory muscle training (IMT), as follows: MIP in GT from $-57.20 \pm 18.76$ to $-79.00 \pm 15.38$ and in GNT from $-52.00 \pm 18.76$ to $-72.66 \pm 19.33$. The prophylactic therapy in the preoperative outpatient provides a significant increase in inspiratory muscle strength in both groups, as evidenced by the increase in MIP with consequent improvement in ventilatory capacity.

Keywords I physical therapy specialty; esophagectomy; smoking.

\footnotetext{
Estudo desenvolvido na Universidade Federal do Triângulo Mineiro (UFTM) - Uberaba (MG), Brasil. 'Fisioterapeuta, Pós-Doutoranda em Ciências pela UFTM - Uberaba (MG), Brasil.

${ }^{2}$ Fisioterapeuta, Doutora em Ciências da Saúde pela Universidade Federal de São Paulo (UNIFESP) - São Paulo (SP), Brasil. ${ }^{3}$ Professor-Titular da Disciplina de Cirurgia do Aparelho Digestivo na UFTM - Uberaba (MG), Brasil.
} 


\section{INTRODUÇÃO}

O uso do tabaco associado às agressões cirúrgicas pode alterar a mecânica respiratória, o padrão respiratório, as trocas gasosas e os mecanismos de defesa pulmonar, favorecendo a ocorrência de atelectasias, infecções traqueobrônquicas, pneumonias e insuficiência respiratória $\operatorname{aguda}^{1}$. A alteração respiratória mais comum é a fraqueza muscular, que pode levar à diminuição da capacidade vital $(\mathrm{CV})$ e da capacidade inspiratória $(\mathrm{CI})$, evoluindo para distúrbios restritivos, causadores de microatelectasias, hipoventilação alveolar crônica, pneumonias recorrentes e insuficiência respiratória ${ }^{2}$.

Pacientes tabagistas submetidos à fisioterapia respiratória no pré- e pós-operatório de gastroplastia apresentaram declínio maior da pressão inspiratória máxima (PImáx) e da pressão expiratória máxima (PEmáx) no pós-operatório, quando comparados ao grupo de pacientes não tabagistas ${ }^{3}$. Esses resultados mostram a importância do treinamento muscular inspiratório (TMI) para pacientes tabagistas no pré-operatório ambulatorial, pois tem como objetivo melhorar a força muscular e a capacidade funcional respiratória, evitando complicações que aumentem a permanência desses pacientes no hospital.

Desse modo, o reconhecimento dos efeitos nocivos do tabaco motivou a realização deste estudo comparativo, dedicado a avaliar a eficácia do TMI em pacientes tabagistas e não tabagistas que seriam submetidos à cirurgia do megaesôfago, identificando se o fato de ser tabagista influenciaria ou não no ganho de força muscular.

\section{MATERIAIS E MÉTODOS}

Foi realizado um estudo prospectivo experimental de coorte com abordagem quali-quantitativa no Ambulatório Maria da Glória, da Universidade Federal do Triângulo Mineiro (UFTM), pela disciplina de Cirurgia do Aparelho Digestivo. Primeiramente, a pesquisa foi analisada e aprovada pelo Comitê de Ética em Pesquisa da UFTM (processo $n^{0}$ 660). Além disso, cada paciente forneceu, por escrito, consentimento livre após esclarecimento, conforme a resolução 19696 do Conselho Nacional de Saúde.

Os 17 pacientes maiores de 18 anos analisados sendo 12 do sexo masculino e 5 do feminino -, foram divididos em: grupo tabagista $(n=10)$ e não tabagista $(\mathrm{n}=7)$, sendo a amostra intencional, sem perdas ou exclusões. Todos os participantes eram portadores de megaesôfago e seriam submetidos ao tratamento cirúrgico por meio da esofagectomia subtotal trans-hiatal, realizada pela técnica laparoscópica. Os critérios de exclusão foram: incapacidade para a realização do treinamento muscular respiratório (TMR), presença de doença pulmonar aguda ou crônica e recusa à realização da terapêutica proposta.

Todos os pacientes com diagnóstico de megaesôfago e indicação cirúrgica eram encaminhados para o ambulatório de acompanhamento pré-operatório. $\mathrm{Na}$ consulta inicial ambulatorial, foi realizada avaliação fisioterapêtica, que constava de anamnese e avaliação da força muscular respiratória.

O teste de força muscular respiratória baseia-se nas medidas PImáx e PEmáx, que permitem uma avaliação simples, rápida e reproduzível da força muscular respiratória. As medidas foram obtidas pela leitura em um manovacuômetro graduado de -120 a $+120 \mathrm{cmH}_{2} 0^{4}$ (Comercial Médica ${ }^{\circledR}$, São Paulo, Brasil) com o paciente sentado confortavelmente, utilizando clipe nasal e bocal plástico rígido. $\mathrm{O}$ bocal possuía um pequeno orifício, de aproximadamente 2 $\mathrm{mm}$, para neutralizar a pressão da cavidade oral durante a contração dos músculos faciais. $\mathrm{O}$ paciente foi anteriormente orientado sobre a manobra, ressaltando a importância de evitar o escape de $a^{5}$. Para a medida de PImáx, o participante foi orientado a realizar esforço inspiratório máximo sustentado por, no mínimo, um segundo a partir de uma expiração máxima (volume residual). A PEmáx foi obtida no momento em que o paciente realizou um esforço expiratório máximo sustentado por, no mínimo, um segundo após inspiração profunda, partindo da capacidade pulmonar total $(\mathrm{CPT})^{6}$.

As medidas da PImáx e da PEmáx foram realizadas três vezes consecutivas, com intervalo de um minuto entre elas. $\mathrm{O}$ valor mais alto era registrado, sendo que a diferença entre os valores deveria ser de $10 \%$ ou menos para cada repetição e o último não deveria ser o maior ${ }^{7}$. As quantias eram coletadas semanalmente durante o retorno ao ambulatório pré-operatório do departamento de cirurgia do aparelho digestivo.

Após a avaliação, iniciou-se o TMI, realizado com o auxílio do resistor inspiratório denominado Threshold (Respironics ${ }^{\circledR}$ ), pelo período de quatro semanas. A carga utilizada foi de $50 \%$ do valor da PImáx, sendo esta reavaliada semanalmente para reajuste ${ }^{7}$. O paciente realizava o TMI uma vez por semana (três séries de dez 
repetições), no ambulatório sob a supervisão do fisioterapeuta, sendo orientado e estimulado a fazer os exercícios na própria residência nos demais dias da semana (três séries de dez repetições). Para tal, cada paciente recebeu um aparelho Threshold para uso durante todo o preparo pré-operatório e a orientação de registrar por escrito os horários do TMI realizados em casa.

A distribuição do gênero entre tabagistas e não tabagistas foi comparada pelo teste exato de Fisher. As variáveis numéricas foram inicialmente verificadas quanto à sua normalidade e homogeneidade pelos testes de Kolmogorov-Smirnov e Bartlett, respectivamente. Verificada a normalidade, os dados foram expressos em média \pm desvio-padrão. Os valores de idade e as medidas antropométricas foram comparadas pelo teste $t$ de Student, e os de PImáx e PEmáx, teste $t$ de Student pareado. Diferenças foram consideradas estatisticamente significativas quando $\mathrm{p}<0,05$.

\section{RESULTADOS}

Foram estudados 17 pacientes portadores de megaesôfago, divididos em dois grupos: tabagistas e não tabagistas. O GT era composto por 10 pacientes (58,82\%), sendo 9 homens e 1 mulher com idade média de 52,30 anos (31-66). Já o GNT, apresentava 7 pacientes $(41,18 \%)$, sendo 3 homens e 4 mulheres com idade média de 49,70 anos (31-74). Na Tabela 1 estão as características deles com relação a sexo, idade e variáveis antropométricas.

Observamos diferenças estatisticamente significativas quando comparamos os valores obtidos no momento da avaliação inicial e após quatro semanas de fortalecimento muscular inspiratório em ambos os grupos. O GT apresentou resultados significativos no ganho de força muscular inspiratória, observado entre a avaliação, a terceira e a quarta semana de TMR $(p=0,0099$ e $\mathrm{p}=0,0040$, respectivamente). O GNT registrou aumento significante na análise feita entre a avaliação, a segunda e a terceira semana de TMR ( $p=0,0034$ e $p=0,0062)$.

Quanto à PEmáx, observamos incremento da força muscular expiratória em ambos os grupos após quatro semanas de treinamento, apesar de não termos realizado o fortalecimento dos músculos expiratórios. O GT mostrou crescimento significativo no ganho de força muscular expiratória entre a avaliação, a primeira e a quarta semana de TMR ( $p=0,0531$ e $p=0,0479$, respectivamente). No GNT, ele foi verificado entre a avaliação, a segunda e a quarta semana $(p=0,0165$ e $p=0,0021)$. Na Tabela 2 encontram-se os valores das médias e desvio-padrão dos GT e GNT no início e durante as quatro semanas de fortalecimento muscular inspiratório.

\section{DISCUSSÃO}

Os programas de TMI no pré-operatório têm sido empregados com o propósito de aumentar a força e a resistência pulmonar, e diversos estudos já constataram seus benefícios ${ }^{8-12}$. Em seu estudo, Galvan et al. ${ }^{13}$ relataram que, após duas semanas de fortalecimento muscular inspiratório, houve aumento nos valores da PImáx

Tabela 1. Sexo, idade e variáveis antropométricas dos grupos tabagistas e não tabagistas submetidos ao treinamento muscular respiratório

$\begin{array}{lccc}\text { Variáveis } & \begin{array}{c}\text { Grupo } \\ \text { tabagista }\end{array} & \begin{array}{c}\text { Grupo não } \\ \text { tabagista }\end{array} & \text { Valor } p \\ \text { Sexo } & 9 \mathrm{M} / \mathrm{F} & 3 \mathrm{M} / 4 \mathrm{~F} & \mathrm{NS} \\ \text { Idade } & 52,3 \pm 9,76 & 49,7 \pm 15,2 & \text { NS } \\ \text { Peso }(\mathrm{kg}) & 69,21 \pm 4,53 & 69,93 \pm 8,03 & \text { NS } \\ \text { Altura (m) } & 1,72 \pm 0,04 & 1,69 \pm 0,05 & \text { NS } \\ \text { IMC } & 23,17 \pm 0,89 & 24,24 \pm 1,58 & \text { NS }\end{array}$

M: masculino; F: feminino; NS: não significante; IMC: indice de massa corpórea Comparação entre sexo pelo teste exato de Fisher e das variáveis numéricas expressas em média \pm desvio-padrão pelo teste $t$ de Student

Tabela 2. Média e desvio-padrão da pressão inspiratória máxima e da pressão expiratória máxima dos grupos tabagista e não tabagista no início e durante as quatro semanas de treinamento muscular respiratório

\begin{tabular}{|c|c|c|c|c|c|}
\hline Momento & Inicial & $1^{\circ}$ semana & $2^{\circ}$ semana & $3^{\circ}$ semana & $4^{\circ}$ semana \\
\hline \multicolumn{6}{|l|}{ PImáx } \\
\hline Tabagistas & $-57,20 \pm 18,76$ & $-64,10 \pm 12,86$ & $-70,00 \pm 14,42$ & $-70,00 \pm 14,42^{*}$ & $-79,00 \pm 15,38^{*}$ \\
\hline Não tabagistas & $-52,00 \pm 18,76$ & $-66,85 \pm 22,71$ & $-72,00 \pm 18,18^{*}$ & $-72,00 \pm 18,18^{*}$ & $-72,66 \pm 19,33$ \\
\hline \multicolumn{6}{|l|}{ PEmáx } \\
\hline Tabagistas & $66,00 \pm 24,09$ & $75,20 \pm 18,64^{*}$ & $71,76 \pm 31,08$ & $66,88 \pm 24,68$ & $75,50 \pm 19,05^{*}$ \\
\hline Não tabagistas & $54,28 \pm 14,58$ & $58,28 \pm 17,26$ & $69,14 \pm 23,17^{*}$ & $66,00 \pm 15,70$ & $77,33 \pm 20,34^{*}$ \\
\hline
\end{tabular}

PImáx: pressão inspiratória máxima; PEmáx: pressão expiratória máxima

Variáveis numéricas expressas em média ¿desvio-padrão; Comparações relativas à medida inicial pelo teste $t$ de Student pareado

${ }^{*} \mathrm{p}<0,05$ relativo à medida inicial 
em pacientes tabagistas submetidos ao TMR. Oliveira et al. ${ }^{14}$ observaram que tabagistas que participaram de dez sessões de TMI no pré-operatório apresentaram significativa melhora da PImáx e em sua ventilação voluntária máxima. Na pesquisa de Paisani et al. ${ }^{3}$ foram avaliados pacientes tabagistas (14,2\%), ex-tabagistas $(28,5 \%)$ e não tabagistas $(57,1 \%)$, submetidos à fisioterapia respiratória no pré e pós-operatório de gastroplastia. No pós-operatório verificou-se que no GT houve declínio maior da PImáx, de 51\%, e da PEmáx, de 39\%. Esses dados mostram a importância da intervenção fisioterapêutica precoce em pacientes com fator de risco cirúrgico como o tabagismo.

Após uma metanálise, Weiner et al. ${ }^{15}$ constataram que o TMI foi adequado para melhorar a performance muscular respiratória. Ramirez-Sarmiento et al. ${ }^{16}$ mostraram que o mesmo treinamento induz à melhora funcional e a mudanças adaptativas nas estruturas desses músculos ${ }^{17}$. No presente estudo, ocorreu aumento significativo na força dos músculos inspiratórios em ambos os grupos, evidenciada pelo acréscimo na PImáx. Os resultados sugerem que o ganho de força da musculatura inspiratória proporcionou melhora da capacidade ventilatória, com aumento no volume pulmonar devido à amplitude maior de movimento do tórax e do abdômen durante a inspiração, o que pode ter contribuído para a melhora do desempenho funcional respiratório. Apesar do fortalecimento ter sido empregado apenas para esses músculos, constatamos aumento nos valores da PEmáx em ambos os grupos estudados, confirmando a hipótese de que houve melhora da mecânica respiratória. Os resultados obtidos na pesquisa de Ribeiro et al. ${ }^{18}$ corroboram com os do nosso estudo, pois pacientes submetidos ao TMI e à reeducação respiratória apresentaram melhora significativa também da PEmáx.

Uma pesquisa realizada em pacientes candidatos à cirurgia torácica demonstrou melhoras significativas nas forças musculares respiratórias após reabilitação pulmonar pré-operatória durante um período que variou entre uma e três semanas, de acordo com a internação destes. Tais autores concluíram que a respiração diafragmática vigorosa aumentou a força dos músculos inspiratórios ${ }^{19}$.

O TMI realizado em pacientes com doença pulmonar obstrutiva crônica (DPOC) trouxe um importante benefício a eles com relação à melhora da força muscular inspiratória ${ }^{18}$. Os resultados mostram que mesmo em pessoas com dispneia e deterioração das funções pulmonares, o TMI pode ser eficiente. No presente estudo, o GT não apresentava doenças pulmonares agudas ou crônicas, o que pode ter contribuído para uma boa resposta deles ao TMI.

\section{CONCLUSÃO}

O presente estudo apresentou limitações pelo fato do TMI não ter sido realizado diariamente com a supervisão de um fisioterapeuta. Os pacientes faziam os exercícios apenas uma vez por semana no ambulatório e o restante dos dias em casa, o que impossibilita afirmar que foram concretizados conforme orientação. Talvez, se tivessem sido realizados com a supervisão de um profissional, os resultados seriam ainda mais satisfatórios.

O TMI realizado no pré-operatório ambulatorial foi eficaz, pois aumentou a força muscular inspiratória, incluindo as pessoas que tinham o hábito de fumar. A melhora na força dos músculos respiratórios sugere a utilização do fortalecimento muscular inspiratório na prevenção dos riscos de complicações pulmonares pós-operatória ou o retardo na alta desses pacientes.

Mais estudos são necessários para a melhor elucidação do ganho de força muscular inspiratória no pré-operatório ambulatorial de pacientes tabagistas e nãotabagistas e, então, será possível apontar características que possam contribuir ou não para uma boa resposta desses pacientes ao treinamento.

\section{REFERÊNCIAS}

1. Dudzińska K, Mayzner-Zawadzka E. Tobacco smoking and the perioperative period. Anestezjol Intens Ter. 2008;40(2):108-13.

2. Faria ICB, Abate AK, Rezende IMO, Silva IMM, Avila TRO. Avaliação da capacidade inspiratória em crianças com distrofia muscular progressiva. Fisioter Mov. 2008;21(1):57-63.

3. Paisani DM; Chiavegato LD; Faresin SM. Volumes, capacidades pulmonares e força muscular respiratória no pós-operatório de gastroplastia. J Bras Pneumol. 2005;31(2):125-32.

4. Black LF, Hyatt RE. Maximal respiratory pressures: normal values and relationship to age and sex. Am Rev Respir Dis. 1969;99(5):696-702.

5. Parreira VF, França DC, Zampa CC, Fonseca MM, Tomich GM, Britto RR. Pressões respiratórias máximas: valores encontrados e preditos em indivíduos saudáveis. Rev Bras Fisioter. 2007;11(5):361-8.

6. Souza RB. Pressões respiratórias estáticas máximas. J Pneumol 2002; 28(Suppl 3):S155-65.

7. Neder JA, Andreoni S, Lerario MC, Nery LE. Reference values for lung function tests. II. Maximal respiratory pressures and voluntary ventilation. Braz J Med Biol Res. 1999;32(6):719-27. 
8. Lötters F, van Tol B, Kwakkel G, Gosselink R. Effects of controlled inspiratory muscle training in patients with COPD: a meta-analysis. Eur Respir J. 2002;20(3):570-6.

9. Reeve JC, Nicol K, Stiller K, McPherson KM, Birch P, Gordon IR, et al. Does physiotherapy reduce the incidence of postoperative pulmonary complications following pulmonary resection via open thoracotomy? A preliminary randomised single-blind clinical trial. Eur J Cardiothorac Surg. 2010;37(5):1158-66.

10. Forti E, Ike D, Barbalho-Moulim M, Rasera I Jr, Costa D. Effects of chest physiotherapy on the respiratory function of postoperative gastroplasty patients. Clinics (Sao Paulo). 2009;64(7):683-9.

11. Van't Hul AJ, Postmus PE. Prevention of postoperative pulmonary complications by preoperative training. Ned Tijdschr Geneeskd. 2007:151(45):2491-3.

12. Dronkers J, Veldman A, Hoberg E, van der Waal C, van Meeteren $\mathrm{N}$. Prevention of pulmonary complications after upper abdominal surgery by preoperative intensive inspiratory muscle training: a randomized controlled pilot study. Clin Rehabil. 2008;22(2):134-42.

13. Galvan CC, Cataneo AJ. Effect of respiratory muscle training on pulmonary function in preoperative preparation of tobacco smokers. Acta Cir Bras. 2007;22(2):98-104.
14. Oliveira BV, Wosiacki Filho W, Oliveira A, Pires RA, Mazzaro MA, Romani $J C P$, et al. Fisioterapia pré-operatória em pacientes candidatos à cirurgia por neoplasia de cabeça e pescoço. Rev Bras Cancerol. 1998:44(2):147-54.

15. Weiner P, Magadle R, Beckerman M, Weiner M, Berar-Yanay $N$. Comparison of specific expiratory, inspiratory, and combined muscle training programs in COPD. Chest. 2003:124(4):1357-64.

16. Ramirez-Sarmiento A, Orozco-Levi M, Guell R, Barreiro E, Hernandez $\mathrm{N}$, Mota S, et al. Inspiratory muscle training in patients with chronic obstructive pulmonary disease: structural adaptation and physiologic outcomes. Am J Respir Crit Care Med. 2002:116(11):1491-7.

17. Trevisan ME, Porto AS, Pinheiro TM. Influence of respiratory and lower limb muscle training on functional performance of subjects with COPD. Fisioter Pesq. 2010;17(3):209-13.

18. Ribeiro KP, Toledo A, Whitaker DB, Reyes LC, Costa D. Inspiratory Muscle Training in Rehabilitation of COPD Patients. Saúde Rev. 2007:9(22):39-46

19. Nomori H, Kobayashi R, Fuyuno G, Morinaga S, Yashima $H$. Preoperative respiratory muscle training. Assessment in thoracic surgery patients with special reference to postoperative pulmonary complications. Chest. 1994;105(6):1782-8. 